\title{
Motion Correction in fMRI by Mapping Slice-to-Volume with Concurrent Field-Inhomogeneity Correction
}

\author{
Desmond T.B. Yeo ${ }^{1,2}$, Jeffery A. Fessler ${ }^{2}$, and Boklye Kim ${ }^{1}$ \\ ${ }^{1}$ Department of Radiology, University of Michigan Medical School, MI 48109, USA \\ \{tbyeo, boklyek\} @umich. edu \\ ${ }^{2}$ Department of Electrical Engineering and Computer Science, University of Michigan, \\ MI 48109, USA \\ fessler@umich.edu
}

\begin{abstract}
Head motion is the major source of error in measuring intensity changes related to given stimuli in fMRI. The effects of head motion are image shifts and field inhomogeneity variations which cause local changes in geometric distortions. The previously developed motion correction method, mapping slice-to-volume (MSV), retrospectively remaps slices that are shifted by head motion to their spatially correct locations in an anatomical reference. Images exhibiting spatially varying geometric distortions require non-linear mapping solutions. An accurate field map can be used for the correction of such spatial distortions. However, field-map changes with head motion and, in practice, only one field-map is available typically. This work evaluates the improved motion correction capability of MSV with concurrent iterative fieldcorrected reconstruction using only an initial field-map. The results from simulated motion data show effective convergence and accuracy in image registration for the correction of image artifacts complicated by the motion induced field effects.
\end{abstract}

\section{Introduction}

In fMRI, the voxel intensity differences of echo-planar imaging (EPI) data from the stimulus and rest images, typically in the range of $1 \%$ to $4 \%$, are used to generate an activation map. A major source of signal variation that has adverse effect in accurate measurements of the voxel intensity changes is rigid head motion. EPI technique is sensitive to magnetic susceptibility-induced geometric distortions, especially in the mid to lower brain images. The effect of head motion is not only the artificial linear spatial shifts in the image intensities, but the subsequent local changes in geometric distortions caused by the field inhomogeneity variations induced by the head rotation. Such effects cause the inconsistency of the voxel positions between the images and, consequently, the inaccuracy in statistical testing of the signal changes in response to the given tasks in activation studies.

In multi-slice EPI data, each slice is subject to different motion. Previously, in our group, a realistic motion-correction scheme, mapping a slice to volume (MSV), 
that accounts for inter-slice motion, was developed [1]. It allows individual slices within a volume to be mapped onto an anatomically correct volume reference. The MSV method using rigid-body function has demonstrated a capability to accurately correct image shifts due to the rigid head motion and improved sensitivity and specificity in locating activated regions as compared to the widely used, volume-tovolume registration of EPI volumes which assumes no inter-slice motion, i.e., incorrectly stacked EPI slices [1]. While the rigid-body transform function is sufficient for localizing activations in the sensorimotor cortex, spatial distortions in EPI slices acquired from the mid to lower structures of the brain cause difficulty in localizing activations, i.e., language. Consequently, MSV was expanded to include a non-linear warping function for the studies involving activations in mid brain regions [2][3], however, at a computational cost of longer optimization process associated with higher degrees of freedom (DOF) in registration.

Geometric distortion can be corrected by an accurate field-map which quantifies the deviation of the magnetic field induced by the position of an object in the applied field. Since the head movement causes change in a field map, an accurate geometric distortion correction requires multiple real time field-maps to track the temporal changes in the field-inhomogeneity. This may require modified acquisition sequences to collect field maps simultaneously with each EPI slice by collecting additional k-space data, which may not be available in most scanners, with the increased acquisition time to obtain an adequate resolution in field maps.

In this work, a concurrent motion and field-inhomogeneity correction using a quadratic penalized least squares reconstruction is introduced as an enhancement to the MSV process [1][4]. The method requires only the acquisition of an initial fieldmap. At each iteration, the field map is updated using the motion parameters obtained from MSV. The result demonstrates an improved accuracy in MSV with rigid-body function by incorporating changes in field map to correct image distortions.

\section{Background}

\subsection{EPI Susceptibility-Induced Geometric Distortion}

Geometric distortion is readily observed in the area where local magnetic fieldinhomogeneity is observed, typically at the boundary of two tissues with significant magnetic susceptibility difference. Changing the orientation of the tissue boundary with $B_{0}$ (i.e., out-of-plane rotations) may change the field-map drastically. Translations and in-plane rotations are less likely to change the susceptibilityinduced component of the field-map. In EPI, field-inhomogeneity causes pixels to shift mainly in the phase-encode, i.e., PE, direction [5]. The shift in PE direction, which causes the local geometric distortion, depends on the EPI readout time $T_{\text {readout }}$ and the point field-inhomogeneity $\Delta B\left(x_{i}, y\right)$ as shown in the impulse response

$$
h\left(x_{i}, y\right)=\delta\left(x_{i}, y-\gamma \Delta B\left(x_{i}, y_{1}\right) T_{\text {readout }} \Delta y\right)
$$

where $\gamma$ is the gyromagnetic ratio, $\Delta y$ is the voxel length in the PE direction and $\delta\left(\mathrm{x}_{\mathrm{i}}, \mathrm{y}\right)$ is the input impulse location before distortion. 


\subsection{Map Slice-to-Volume (MSV) Registration}

Statistical analysis of brain activations in fMRI relies on the intensity variation at consistent image voxel locations throughout the time series data. The voxel displacements, in-plane and out-of-plane, associated with the patient's head motion is corrected retrospectively by mapping a slice image onto an anatomically correct reference volume (i.e. map-slice-to-volume, MSV) [1]. The MSV method in this paper allows each slice to have its own six DOF, i.e., rigid-body transform. Automated 3D registration of a slice into an anatomical volume is accomplished by optimizing the mutual information metric. The transformation that gives the lowest MI metric in the iterative optimization scheme is used to compute the final position of a slice in the spatial reference.

\subsection{Iterative Field-Corrected Reconstruction}

Most geometric distortion correction methods assume a smooth field-map [5][6]. We use an iterative field-corrected reconstruction method that does not assume a smooth field-map [4]. The continuous object $f$ and field-map $\Delta \omega$ are parameterized into a sum of weighted rect functions $b\left(\vec{r}-\vec{r}_{n}\right)$. Ignoring spin relaxation and assuming uniform receiver coil sensitivity, the parameterized MR signal equation for a slice is

$$
s\left(t_{i}\right) \approx B\left(\vec{k}\left(t_{i}\right)\right) \sum_{n=0}^{n_{p}-1} f_{n} e^{-j \Delta \omega_{n} t_{i}} e^{-j 2 \pi\left(\vec{k}\left(t_{i}\right) \vec{\vartheta}_{n}\right)}
$$

where $\mathrm{s}\left(\mathrm{t}_{\mathrm{i}}\right)$ is the baseband signal sample at time $\mathrm{t}_{\mathrm{i}}$ during readout, $B\left(\vec{k}\left(t_{i}\right)\right)$ is the Fourier transform of $b\left(\vec{r}-\vec{r}_{n}\right), f_{n}$ and $\Delta \omega_{n}$ are the object intensity and fieldinhomogeneity, respectively, at $\vec{r}_{n}$. The dominant noise in MRI is conventionally modeled as a white Gaussian noise [7]. In matrix form, the sampled signal vector is

$$
y=\mathbf{A} f+\varepsilon
$$

where $f$ and $\varepsilon$ are the column-wise stacked vector of the parameterized object and noise, respectively, $y$ is the $\mathrm{k}$-space data vector and $\mathbf{A}$ is the system-object matrix with elements $a_{m, n}=B\left(\vec{k}\left(t_{m}\right)\right) e^{-j \Delta \omega_{n} t_{m}} e^{-j 2 \pi\left(\vec{k}\left(t_{m}\right) \boldsymbol{\bullet}_{r_{n}}\right)}$. The object $f$ is estimated directly from the k-space data $y$ by minimizing a quadratic penalized least squares (QPLS) cost function using the conjugate gradient optimization algorithm in conjunction with time-segmentation and min-max interpolation. The cost function and estimator are

$$
\begin{gathered}
\psi_{1}(f)=\frac{1}{2}\|y-\mathbf{A} f\|^{2}+\frac{1}{2} \beta f^{T} \mathbf{C}^{\top} \mathbf{C} f \\
\hat{f}_{Q P L S}=\underset{f}{\arg \min } \psi_{1}(f)=\left[A^{*} A+\beta R\right]^{-1} A^{*}[A f+\varepsilon]
\end{gathered}
$$

where $\mathbf{C}$ is a $\mathrm{n}_{\mathrm{p}}-1 \times \mathrm{n}_{\mathrm{p}}$ second order differencing matrix. 


\section{Methods}

\subsection{Concurrent Motion and Field-Inhomogeneity Correction Scheme}

The concurrent correction scheme is summarized in Fig. 1. The key idea is to use the rigid-body motion parameter estimates from the MSV process to transform the original field-map into an updated field-map volume. This new field-map is then used to reconstruct the original EPI slices using the iterative QPLS method. The reconstructed EPI slices are registered with rigid-body MSV to obtain a new set of motion parameters. The algorithm repeats until good estimates are obtained. Estimation error propagation is minimized by using the original data in each cycle.

A set of geometrically distorted EPI images with motion are simulated from a $\mathrm{T}_{2}$-weighted volume from the International Consortium of Brain Mapping (ICBM) such that ground truths exist for each slice's motion parameters and its non-distorted form. A simulated field-map is used for the forward distortion. The concurrent correction scheme is then applied to the simulated EPI images and evaluated in terms of its ability to recover the true motion parameters and the true non-distorted images.

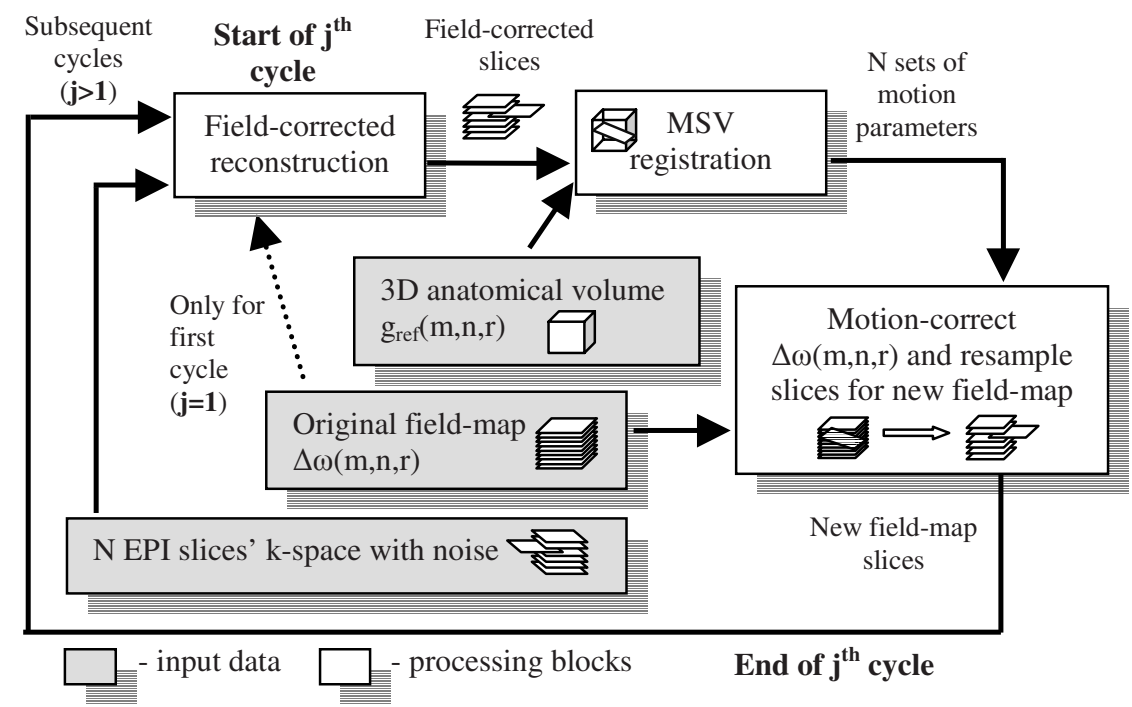

Fig. 1. Concurrent motion and field-inhomogeneity correction scheme.

\subsection{Motion and Distortion Simulation}

Two anatomically correct $\mathrm{T}_{1^{-}}$and $\mathrm{T}_{2}$-weighted image datasets from the ICBM are used for this motion correction experiment. The two volumes, in $256 \times 256 \times 181$ matrix with a voxel size of $1 \mathrm{~mm}^{3}$, are originally in perfect registration. The $\mathrm{T}_{2}-\mathrm{W}$ volume is used to simulate the geometrically distorted EPI data with motion as shown in Fig. 2. The ICBM T $\mathrm{T}_{2}-\mathrm{W}$ volume is resampled in the slice direction to make the slice thickness $5 \mathrm{~mm}$; EPI slices are typically $3 \mathrm{~mm}$ to $6 \mathrm{~mm}$ thick. For simplicity, only 
the rigid body motion parameters $\mathrm{t}_{\mathrm{x}}, \mathrm{t}_{\mathrm{y}}, \mathrm{t}_{\mathrm{z}}$ (translation) and $\theta_{\mathrm{z}}$ (in-plane rotation) are applied to the $\mathrm{T}_{2}-\mathrm{w}$ volume.

The field-inhomogeneity is simulated at a level comparable to realistic values. The maximum simulated field-inhomogeneity is $8 \mathrm{ppm}$ at $1.5 \mathrm{~T}$, which is close to the air-tissue field-inhomogeneity range of $\approx 9 \mathrm{ppm}$. The range of applied motion was intentionally large with maximum values of $7.85 \mathrm{~mm}, 7.85 \mathrm{~mm}, 10.2 \mathrm{~mm}$ and $7.85^{\circ}$ for $\mathrm{t}_{\mathrm{x}}, \mathrm{t}_{\mathrm{y}}, \mathrm{t}_{\mathrm{z}}$ and $\theta_{\mathrm{z}}$, respectively. The motion applied is smooth with respect to time as the head does not typically make sudden movements. Rotation about the z-axis and all translations do not change the orientation of the air-tissue interface with respect to $\mathrm{B}_{0}$ and thus is unlikely to change the field-map except for the respective linear translation or in-plane rotation. Thus, forward distorting the $T_{2}$ volume with the rotatedtranslated field-map is reasonable as long as out-of-plane rotations $\theta_{\mathrm{x}}$ and $\theta_{\mathrm{y}}$ are not applied.

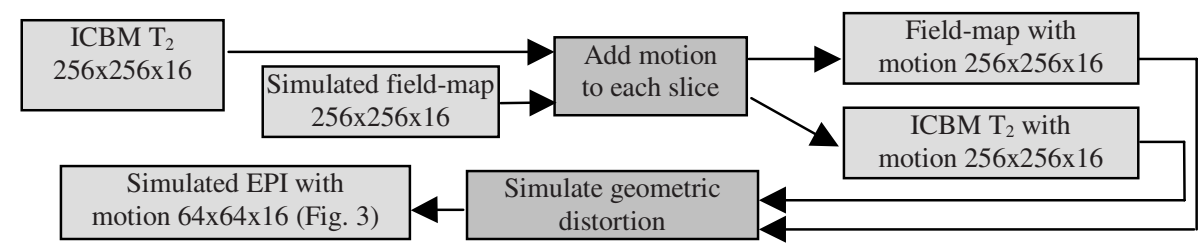

Fig. 2. Simulating the motion and geometric distortion using a synthetic field map applied to the $\mathrm{T}_{2}-\mathrm{w}$ ICBM images.

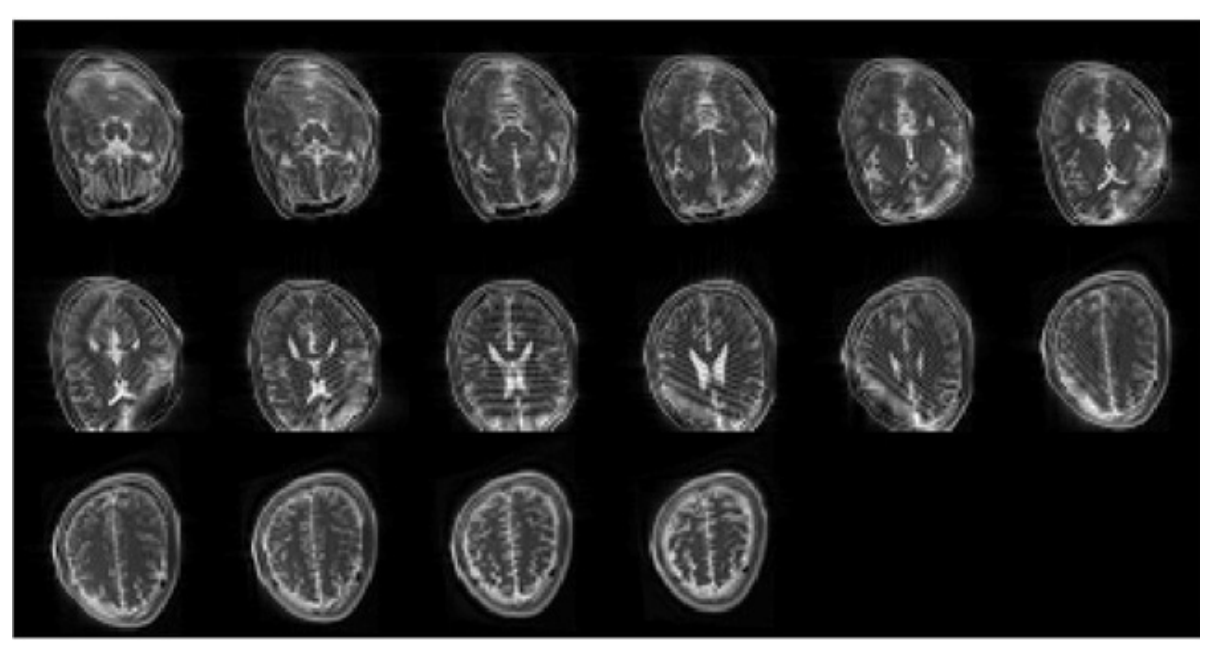

Fig. 3. Simulated images with geometric distortion representing EPI data with motion artifacts. 


\subsection{Field-Map Update}

The set of MSV-recovered registration parameters for each slice is applied to the field-map which is then resampled at the appropriate slice locations to obtain the updated field-map for the next cycle. In the first cycle, field-corrected reconstruction uses the original field-map, which is inaccurate because the head has moved since the time of acquisition. It is hypothesized that not all the 6 motion parameters should be used in the initial cycle(s) to compute the updated field-map as some are unreliable estimates when the field-map is initially not correct. Since any error in the field-map will likely manifest itself as a larger MSV registration error in the phase-encoded direction, $\mathrm{t}_{\mathrm{y}}$ is not a reliable parameter for updating the field-map in the initial cycle(s). The out-of-plane rotation parameters $\theta_{\mathrm{x}}$ and $\theta_{\mathrm{y}}$ may change the field-map significantly and thus are also not deemed to be reliable in the early cycles. In the first cycle, only $t_{x}, t_{z}$ and $\theta_{z}$ are used to update the field-map. In the second and third cycles, $\mathrm{t}_{\mathrm{x}}, \mathrm{t}_{\mathrm{y}}, \mathrm{t}_{\mathrm{z}}$ and $\theta_{\mathrm{z}}$ are used to update the field-map.

\section{Results}

Figure 4 shows the absolute error or $\left|\boldsymbol{\theta}_{l}-\boldsymbol{\theta}_{l, \text { round truth }}\right|$ at different stages in the proposed correction scheme. $\boldsymbol{\theta}_{l}$ is the MSV-recovered rigid-body motion parameter vector for slice $l$ and $\boldsymbol{\theta}_{l, \text { round truth }}$ is the applied ground truth motion parameter vector. Table 1 lists the RMS error of the data plotted in Fig. 4. In cycle one, the inaccurate original field-map was used to perform the initial field-corrected reconstructions. These cycle1-reconstructed images yield lower RMS in recovery error for all motion parameters compared to the distorted simulated EPI volume. Upon updating the fieldmap with the cycle1 MSV rigid motion parameters, $t_{x}, t_{z}$ and $\theta_{z}$, and performing the field-corrected reconstructions again, the RMS MSV-recovery error for the second cycle is further reduced to a level that is comparable to the experimental ground truth. A third cycle of the proposed scheme is then performed using all the MSV motion parameters from cycle two (except $\theta_{\mathrm{x}}$ and $\theta_{\mathrm{y}}$ ) to update the field-map. The RMSE values for the third cycle remain close to the experimental ground truth, which suggests that convergence has occurred experimentally for the proposed scheme under the applied conditions. The experimental ground truth is the RMS error obtained when registering the $\mathrm{T}_{2}-\mathrm{W}$ volume with simulated motion without geometric distortion to the $\mathrm{T}_{1}$ anatomical volume. The first cycle RMS error can be viewed as the performance of the MSV with rigid-body transform function and fieldinhomogeneity scheme where the two problems are corrected separately. Nevertheless, the RMS errors in the third cycle in table 1 shows improved average performance of over $3 \mathrm{~mm}$ for translations and over 4 degrees for rotations.

Next, the reconstructed image quality at various stages of the proposed correction scheme is compared. Figure 5 shows plots of the normalized RMS error (NRMSE) for each slice using the non-distorted ground truth images with motion as reference images. Compared to the first cycle, the images reconstructed in the second and third cycles have much lower NRMSE values for almost all slices. To provide a performance benchmark, reconstruction is performed using the actual field-map that 
was used to forward distort the $\mathrm{T}_{2}-\mathrm{w}$ volume. Theoretically, these reconstructed images should have the lowest NRMSE values compared to all the previously corrected images. However, due to reconstruction errors, they serve only as an estimate of the best image quality performance achievable. The NRMSE values for the second and third cycles are comparable to each other and to the benchmark, which again suggests that the proposed scheme is experimentally stable under the applied conditions. In summary, the proposed scheme improves both the rigid motion parameters estimates as well as the final reconstructed image quality.
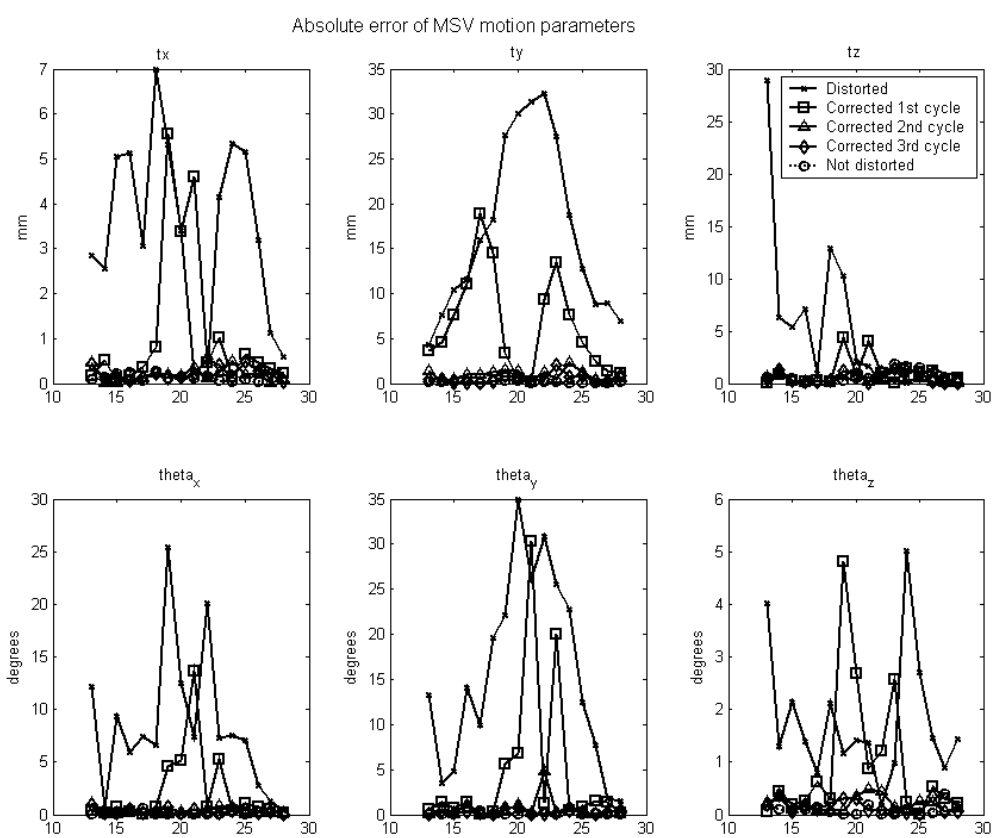

Fig. 4. MSV motion parameters absolute error at different stages in concurrent correction

Table 1. RMS error of MSV-recovered motion parameters over all slices. The ground truth is obtained from registering the $\mathrm{T}_{2}$ - $\mathrm{W}$ slices with simulated motion without geometric distortion to the $\mathrm{T}_{1}$ anatomical volume.

\begin{tabular}{|l|l|l|l|l|l|l|}
\hline \multirow{2}{*}{ Dataset } & \multicolumn{5}{|c|}{ RMS error over all slices } \\
\cline { 2 - 7 } & $\mathbf{t}_{\mathbf{x}}(\mathbf{m m})$ & $\mathbf{t}_{\mathbf{y}}(\mathbf{m m})$ & $\mathbf{t}_{\mathbf{z}}(\mathbf{m m})$ & $\boldsymbol{\theta}_{\mathbf{x}}\left({ }^{\circ}\right)$ & $\boldsymbol{\theta}_{\mathbf{y}}\left({ }^{\circ}\right)$ & $\boldsymbol{\theta}_{\mathbf{z}}\left({ }^{\circ}\right)$ \\
\hline Distorted EPI with motion & 3.95 & 19.48 & 8.80 & 10.61 & 18.75 & 2.14 \\
\hline Corrected 1 $^{\text {st }}$ cycle & $\mathbf{2 . 0 4}$ & $\mathbf{8 . 4 8}$ & $\mathbf{1 . 6 3}$ & $\mathbf{4 . 0 8}$ & $\mathbf{9 . 3 8}$ & $\mathbf{1 . 5 9}$ \\
\hline Corrected 2 $^{\text {nd }}$ cycle & $\mathbf{0 . 2 4}$ & $\mathbf{1 . 0 8}$ & $\mathbf{0 . 6 3}$ & $\mathbf{0 . 4 5}$ & $\mathbf{1 . 3 2}$ & $\mathbf{0 . 2 5}$ \\
\hline Corrected 3 $^{\text {rd }}$ cycle & $\mathbf{0 . 2 8}$ & $\mathbf{0 . 7 4}$ & $\mathbf{0 . 7 8}$ & $\mathbf{0 . 4 2}$ & $\mathbf{0 . 3 8}$ & $\mathbf{0 . 1 9}$ \\
\hline Ground truth & 0.17 & 0.20 & 0.89 & 0.23 & 0.29 & 0.14 \\
\hline
\end{tabular}




\section{Discussion and Conclusions}

A field inhomogeneity correction method using an iterative quadratic penalized least squares reconstruction technique was implemented as a part of MSV motion correction. The motion induced field variation is updated concurrently with the MSV rigid-body transform vectors. The convergence and performance of the concurrent method were evaluated using simulated data to determine the accuracy in registration. Applying the method to motion simulated synthetic phantom data warrants the accurate evaluation of the mapping results with the known ground truths. The results in Table 1, Figs. 4 and 5 demonstrate the effective corrections of the motion artifacts that are complicated by the field effects induced by rigid head motion. The ground truth NRMSE in Fig. 5 is non-zero because of reconstruction errors. Future work will include a study of the tolerance in the range of out-of-plane motion for the correction of human EPI data as well as the validation of the robustness with a phantom.

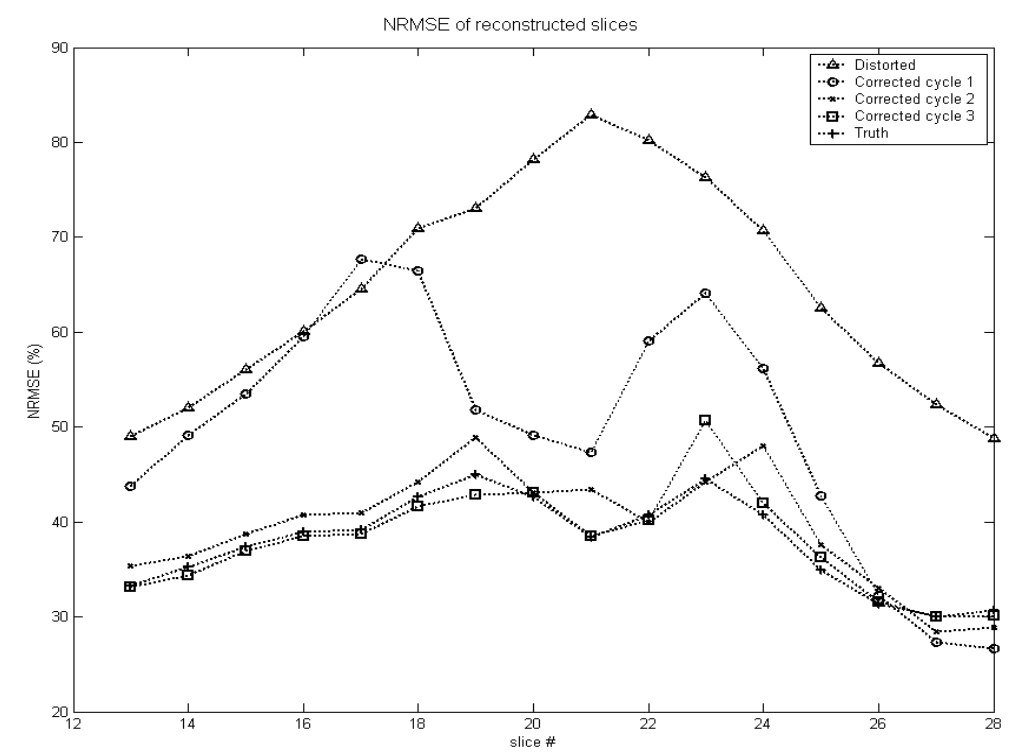

Fig. 5. Normalized RMS error (NRMSE) of each slice

\section{References}

1. Kim, B., J.L. Boes, P.H. Bland, T.L. Chenevert, C.R. Meyer, Motion correction in fMRI via registration of individual slices into an anatomical volume, Magnetic Resonance in Medicine, 41(4), 964-972, 1999

2. Kim, B., P.H. Bland, C.R. Meyer, Correction of local deformations in fMRI by 3D nonlinear warping in map-slice-to-volume approach, Proc. Seventh Annual Meeting of ISMRM, p. 1765, 2000

3. Kim, B, Chenevert, TL, Meyer, CR, Motion correction with a non-linear warping solution for activations in temporal region, Proc. Tenth Annual Meeting of ISMRM, p. 2304, 2002 
4. Bradley P. Sutton, Douglas C. Noll, Jeffrey A. Fessler, Fast, iterative image reconstruction for MRI in the presence of field inhomogeneities, IEEE Transactions on Medical Imaging, Vol. 22, No.2, 178-188, February 2003

5. Peter Jezzard, Robert S. Balaban, Correction for geometric distortion in echo planar images from B0 field variations, Magnetic Resonance in Medicine, 34, 65-73, 1995

6. Rhodri Cusack, Matthew Brett, Katja Osswald, An evaluation of the use of magnetic field maps to undistort echo-planar images, Neuroimage, 18, 127-142, 2003

7. E.M. Haacke, R.W. Brown, M.R. Thomson, R. Venkatesan, Magnetic resonance imaging: physical principles and sequence design, New York, 1999, John Wiley and Sons 